\title{
Tracking of electrochemical impedance of batteries
}

\author{
H.Piret ${ }^{\mathrm{a}}$, P. Granjon ${ }^{\mathrm{b}}$, N. Guillet ${ }^{\mathrm{c}}$, V. Cattin $^{\mathrm{a}}$ \\ ${ }^{a}$ CEA, LETI, MINATEC Campus, F-38054 Grenoble, France, \\ helene.piret@cea.fr, + 33 (0)438785557 \\ ${ }^{b}$ Univ. Grenoble Alpes, GIPSA-Lab, F-38000 Grenoble France \\ ${ }^{c}$ Univ. Grenoble Alpes, INES, F-73375 Le Bourget du Lac, France \\ CEA, LITEN, F-38054 Grenoble, France
}

\begin{abstract}
This paper presents an evolutionary battery impedance estimation method, which can be easily embedded in vehicles or nomad devices. The proposed method not only allows an accurate frequency impedance estimation, but also a tracking of its temporal evolution contrary to classical electrochemical impedance spectroscopy methods. Taking into account constraints of cost and complexity, we propose to use the existing electronics of current control to perform a frequency evolutionary estimation of the electrochemical impedance. The developed method uses a simple wideband input signal, and relies on a recursive local average of Fourier transforms. The averaging is controlled by a single parameter, managing a trade-off between tracking and estimation performance. This normalized parameter allows to correctly adapt the behavior of the proposed estimator to the variations of the impedance. The advantage of the proposed method is twofold: the method is easy to embed into a simple electronic circuit, and the battery impedance estimator is evolutionary. The ability of the method to monitor the impedance over time is demonstrated on a simulator, and on a real Lithium ion battery, on which a repeatability study is carried out. The experiments reveal good tracking results, and estimation performance as accurate as the usual laboratory approaches.

Keywords: battery monitoring system; electrochemical impedance spectroscopy; frequency domain estimation; wideband identification; recursive estimation algorithm.
\end{abstract}




\section{Introduction}

The recent and future expansion of electric vehicles or nomad devices inevitably leads to the development of efficient battery management systems (BMS). Such systems must continuously determine the state of the monitored

5 battery from several measurements. However, in order to preserve the battery integrity, only non invasive and non destructive measurement methods are used, and most BMS measure external quantities such as the current flowing through the battery, the voltage across its terminals and its surface temperature [1] [2]. From these measurements, one way to obtain interesting information representative of the current state of the battery is to estimate its electrochemical impedance 3 . Indeed this quantity describes the dynamic behavior of the battery and regularly changes with the evolution of its internal temperature, state of charge (SoC) and state of health (SoH) 4]. Consequently, the electrochemical impedance is used in many methods to estimate the internal temperature [5] [6] 15 [7, the $\mathrm{SoC}$ [8] 9] [10] and the $\mathrm{SoH}$ [10] [11] of the monitored battery. Usual procedures used to estimate a battery electrochemical impedance belong to the class of active identification methods [12]: known variations are added to the battery input, and the corresponding output variations are measured and used to estimate the unknown impedance on a given frequency band. In the rest of this paper, the battery impedance is estimated in galvanostatic mode: the current $i(t)$ flowing through the battery is considered as the input and the voltage $u(t)$ across the battery terminals as the output. This choice can be justified by the fact that the current can be easily driven by very simple and cheap electronic devices (such as a transistor for example), which is a strong requirement for embedded systems for which this work is developed. Active identification methods rely on two main assumptions:

- Firstly, variations of the additional current used to estimate the impedance are chosen sufficiently small for the battery to have a linear behavior with respect to these variations. Under this assumption, the battery can be considered as a linear system. 
- Secondly, parameters on which the battery characteristics depend are assumed to remain constant during the measurement process. Under this assumption, the battery can be considered as a time-invariant system during the measurement time.

35 Jointly, these two assumptions allow to consider the battery as a linear and time-invariant (LTI) system regarding the additive input-output variations and during the measurement time. In that case, the battery admits a well defined frequency response function, corresponding to its electrochemical impedance $Z(f)$ and verifying the following frequency relationship:

$$
Z(f)=\frac{S_{u i}(f)}{S_{i i}(f)} \text { if } S_{i i}(f) \neq 0 .
$$

In this equation, $S_{u i}(f)$ is the cross power spectral density (CPSD) between voltage and current variations, while $S_{i i}(f)$ is the power spectral density (PSD) of current variations only [13, 14]. Eq. (1) highlights the fact that $Z(f)$ can only be estimated in the frequency bands where the input current variations contain 45 power, i.e. where their PSD is different from 0.

The validity of the LTI assumption for the battery and equivalently the validity of Eq. (1) can be checked by using the notion of magnitude squared spectral coherence [15] defined as:

$$
C_{u i}(f)=\frac{\left|S_{u i}(f)\right|^{2}}{S_{u u}(f) S_{i i}(f)}
$$

${ }_{50}$ where $S_{u u}(f)$ is the PSD of voltage variations.

This frequency domain function is a statistical quantity normalized between 0 and 1 , that can be interpreted as the magnitude squared correlation coefficient between the spectral components of the voltage and the current around a given frequency $f$. It gives a normalized measurement of how linearly the spectral components of these two signals are related to each other. It has been shown for example in [15] that in case of low measurement noise, the identified system can be considered as LTI in frequency bands where the magnitude squared coherence is close to 1 , while the LTI assumption can be rejected in frequency bands where it remains close to 0 . This quantity has been used to check the 
LTI assumption for batteries in [16.

Several methods have been developed to estimate the impedance, such as the time domain step response. The disadvantage of this approach is related to the measured response to an impulse or step input that often have a small amplitude in comparison to the noise, especially when the battery impedance is low. That

65 is why this technique requires extra-large inputs to reach good signal to noise ratios and finally good estimation performance. These large inputs often induce non-linear behavior, explaining why this approach is not used in this study. One of the authoritative methods for battery impedance measurements is the narrowband electrochemical impedance spectroscopy (FFT-EIS) [17] 18. In this method, a single sine wave with low amplitude and fixed frequency is used as input signal. Eq. (1) is then valid at the sine frequency only, and $Z(f)$ can be estimated at that particular frequency only, justifying the term "narrowband". If $Z(f)$ must be estimated for several frequencies, the same measurement process has to be sequentially done for each desired frequency. An efficient way to avoid this sequential implementation and estimate the impedance for a discrete set of frequencies at one time is to use a multisine approach [14. In that case, the input signal consists of a sum of sines which frequencies correspond to the desired set.

For wideband method, input signals are wideband in the sense that their PSD so is different from 0 on a continuous frequency band. In that case, Eq. (1) is valid all over that frequency band, where $Z(f)$ can be estimated whatever $f$. Several options are available to choose a wideband input signal for system identification, the most popular being swept sines, random noises and pseudorandom binary sequences (PRBS) [14].

${ }_{85}$ Eq. (1) and (2) clearly show that the estimation of $Z(f)$ relies exclusively on basic spectral quantities such as PSD and CPSD. A simple and efficient estimator usually used for such quantities is the Welch modified periodogram [19]. The signals are first divided into $L$ consecutive blocks of same length by using a time window. The discrete Fourier transform (DFT) of each block of data is then computed by using a fast Fourier transform algorithm. Finally, the $L$ obtained 
DFTs are multiplied, averaged, and normalized correctly to obtain the desired result. As an example, Eq. (3) gives the expression of the voltage-current CPSD estimator $\hat{S}_{u i}(f)$ :

$$
\begin{aligned}
\hat{P}_{u i_{k}}(f) & =A U_{k}(f) I_{k}{ }^{*}(f), \\
\hat{S}_{u i}(f) & =\frac{1}{L} \sum_{k=0}^{L-1} \hat{P}_{u i_{k}}(f),
\end{aligned}
$$

where $A$ is a normalization factor, ${ }^{*}$ denotes complex conjugation, and $U_{k}(f)$ $\left(I_{k}(f)\right.$ respectively) is the DFT of the $k^{t h}$ block of voltage (current respectively) signal.

In Eq. (3a), $\hat{P}_{u i_{k}}(f)$ is the cross-periodogram of the $k^{\text {th }}$ blocks of voltage and current signals, and Eq. (3b) clearly shows that the estimated CPSD is given by an arithmetic averaging of the $L$ cross-periodograms obtained from the acquired data. Obviously, same type of estimators can be obtained for the current and voltage PSDs $\hat{S}_{i i}(f)$ and $\hat{S}_{u u}(f)$ by using exclusively $U_{k}(f)$ or $I_{k}(f)$ in Eq. (3).

A simple impedance estimator $\hat{Z}(f)$ is obtained by using Eq. (3) in the impedance definition given by Eq. (1).

$$
\hat{Z}(f)=\frac{\hat{S}_{u i}(f)}{\hat{S}_{i i}(f)} \text { where } \hat{S}_{i i}(f) \neq 0 .
$$

Following the same principle, Eq. (3) used in Eq. (2) leads to the estimator of the magnitude squared spectral coherence between battery voltage and current:

$$
\hat{C}_{u i}(f)=\frac{\left|\hat{S}_{u i}(f)\right|^{2}}{\hat{S}_{u u}(f) \hat{S}_{i i}(f)} .
$$

Therefore, Eq. (3), (4) and (5) form together the battery identification algorithm:

- Eq. (3) and (4) give access to the battery impedance estimate in the frequency band of interest,

- Eq. (3) and (5) can be used to check the validity of the LTI assumption for the battery behavior, or equivalently the validity of the estimated impedance. 
The performance of this identification strategy has been studied in numerous general books such as [12, 14] and [15, and in more specific works such as [16] 20] for battery impedance estimation. It has been shown that on the one hand, the variance of the impedance estimator is inversely proportional to the number $L$ of averaged periodograms in Eq (3), which is a quite intuitive result. On the other hand, this variance is also proportional to $\frac{1-C_{u i}(f)}{C_{u i}(f)}$ showing that the best estimation performance will be reached when $C_{u i}(f)$ is close to 1 , that is for true LTI systems and small measurement noises.

While single sine and multisine FFT-EIS reach very accurate estimation performance [14, the set of frequencies at which $Z(f)$ is estimated depends on 130 the chosen input signal. In other words, each time $Z(f)$ must be estimated at slightly different frequencies, a new input signal must be designed and the whole measurement process must be repeated. Moreover, the generation of sine waves with different frequency or multisine signals requires advanced electronic generators. These disadvantages concern both EIS based on phase sensitive detector 21] and FFT-EIS. Even if the generation of input signals is much easier for square current EIS [22], this method only allows to estimate the impedance for a small discrete set of frequency: the fundamental frequency of the square wave and its odd harmonics.

Wideband methods relying on PRBS signals are more suitable for embedded systems. Indeed, such two-level signals can be generated with very simple electronic circuits. PRBS signals reach good estimation performance for electrochemical impedance of batteries [16, although it leads to slightly less accurate results than multisine signals [14. Moreover, the same measurement data set can be used to estimate $Z(f)$ whatever $f$ in the frequency band of the chosen input signal.

However, a joint property of these identification strategies limits their application to embedded systems: they only lead to one estimate of $Z(f)$ per measurement. Consequently, each time a new estimate of $Z(f)$ is needed, the 
to follow the time evolution of $Z(f)$ that can be significant in case of strongly varying operating conditions.

The method detailed in the next section is able to efficiently track the variations of $Z(f)$ over time thanks to a recursive implementation of the wideband approach.

In this context, this work aims at developing a method dedicated to battery electrochemical impedance estimation and tracking, which is sufficiently efficient to precisely track the temporal variations of this quantity, and suitable to be embedded in a vehicle or a nomad device. The proposed method and the corresponding results are presented in this paper with the following organization. Section 2 presents the proposed algorithm based on a local averaging strategy recursively implemented in the frequency domain, and the influence of its main parameters. This algorithm is tested on a simulator and on an experiment conducted on a real battery. The section 3 describes the protocol used in each case. The section 4 details and discusses both the simulate and experimental results.

\section{General principle}

\subsection{Time-varying impedance spectroscopy}

The goal of this study is to obtain a time-dependent estimator of the battery electrochemical impedance. This estimator must be sufficiently accurate to give exact estimates of $Z(f)$, but must also have tracking capabilities to follow its eventual variations over time. One possibility is to use the wideband principles previously detailed, and adapt them to the time-varying case. In the wideband approach, the impedance estimation entirely relies on the Welch mod175 ified periodogram summarized in Eq. (3). More particularly, Eq. (3b) shows that this spectral estimator is based on a global average of the whole set of pediodograms obtained from the acquired data. The main idea behind the proposed method is to replace this global average with a local average, leading 
to time-dependent estimators for the different spectral quantities, and consestrategies are detailed and compared.

\subsection{Local averaging strategies}

Among all averaging strategies, the ones that can be implemented recursively are particularly suitable for embedded systems. In this section, only the two simplest ones are detailed and compared: sliding window averaging, and exponential averaging.

The time-varying spectral estimator obtained with a sliding window averaging consists in the average of the $N$ last periodograms. Following notations used in Eq. (3), the corresponding estimator for the voltage-current CPSD around the block of data number $k$ is given by:

$$
\begin{aligned}
\hat{S}_{u i_{k}}(f) & =\frac{1}{N} \sum_{n=k-N+1}^{k} \hat{P}_{u i_{n}}(f) \\
& =\hat{S}_{u i_{k-1}}(f)+\frac{\hat{P}_{u i_{k}}(f)-\hat{P}_{u i_{k-N}}(f)}{N}
\end{aligned}
$$

where the number of averaged periodograms $N$ is smallest than the total number of periodograms L. Eq. 6b corresponds to the recursive implementation of this estimator, which is more suitable for embedded systems. Obviously, the averaging effect increases with the size of the sliding window $N$.

The spectral estimator obtained with an exponential averaging is given by the following relation:

$$
\hat{S}_{u i_{k}}(f)=\alpha \hat{S}_{u i_{k-1}}(f)+(1-\alpha) \hat{P}_{u i_{k}}(f)
$$

where $\alpha \in[0,1$ [ is usually called the forgetting factor. Eq. 77] clearly shows that when $\alpha=0$, the voltage-current CPSD estimate for block $k \hat{S}_{u i_{k}}(f)$ is simply the cross-periodogram of the corresponding data blocks $\hat{P}_{u i_{k}}(f)$. In that case, no average is performed, leading to poor estimation performance regarding measurement noise. On the contrary, the estimated CPSD is more and more averaged when $\alpha$ increases and tends to 1 . 
As noticed for Eq. (3), similar time-varying estimates are obtained for PSDs by replacing cross-periodograms with periodograms in Eq. (6) and (7).

Two different criteria are used to compare the previous strategies: their estimation performance, and their implementation complexity.

Estimation performance can be easily studied by considering each strategy as a digital filter. Indeed, Eq. (6b) corresponds to the recursive equation of a causal finite impulse response (FIR) filter of order $N$ with all weights equal to $\frac{1}{N}$, while Eq. (7) is the recursive equation of a causal infinite impulse response (IIR) filter of order 1 with one pole $\alpha$. Then, the following filter properties can be used to characterize the performance of each strategy:

- Response time at $r \%$ : this quantity is the number of samples from which the step response of the considered filter reaches $r \%$ of it's final value. In the present context, it can be interpreted as the convergence time at $r \%$ of the averaging strategy.

- Equivalent noise bandwidth: this quantity corresponds to the variance (i.e. the amount of residual fluctuations) at the filter output when supplied with a white noise of variance 1 . For lowpass filters as in this study, it represents the significance of the averaging effect realized by the filter: strong averaging effect for values close to 0 , and weak averaging effect for values close to one.

The values of these characteristics are determined for each averaging strategy by using the weights of the corresponding filter, and are given in Table. 1. Obviously, these characteristics exclusively depend on parameters $N$ and $\alpha$, and show that the two strategies can reach similar estimation performance depending on the values of these two independent parameters. These results are consequently not useful to determine the best strategy, but give interesting links between $N$ and $\alpha$. For example, $\alpha$ can be set such that Eq. (7) has the same averaging effect as Eq. (6). In that case, the two averaging strategies must have the same 


\begin{tabular}{|c|c|c|}
\hline averaging strategy & $\begin{array}{c}\text { sliding window } \\
\text { (FIR filter) }\end{array}$ & $\begin{array}{c}\text { exponential } \\
\text { (IIR filter) }\end{array}$ \\
\hline response time at $r \%$ & $N \frac{r}{100}-1$ & $\frac{\ln \left(1-\frac{r}{100}\right)}{\ln (\alpha)}-1$ \\
\hline equivalent noise bandwith & $\frac{1}{N}$ & $\frac{1-\alpha}{1+\alpha}$ \\
\hline
\end{tabular}

Table 1: Main characteristics of the considered averaging strategies.

\subsection{Time-varying impedance estimator}

As previously mentioned, the global structure of the proposed algorithm is

the same as for the wideband approach, and is described in Fig. 1.

The algorithm complexity can be directly deduced from the recursive equations (6b) and (7). The implementation of these two equations leads to a similar number of operations, but the sliding window approach clearly needs a larger amount of memory. Indeed, in Eq. (7) only two values must be stored to update the spectral estimator whatever the value of $\alpha$. On the contrary, the memory depth needed for Eq. $6 \mathrm{~b}$ is $N+2$, leading to a large amount of memory for large $N$. Therefore, the exponential averaging is much more interesting for embedded systems regarding this second criterion, which justifies the choice of this strategy in the final estimator. 


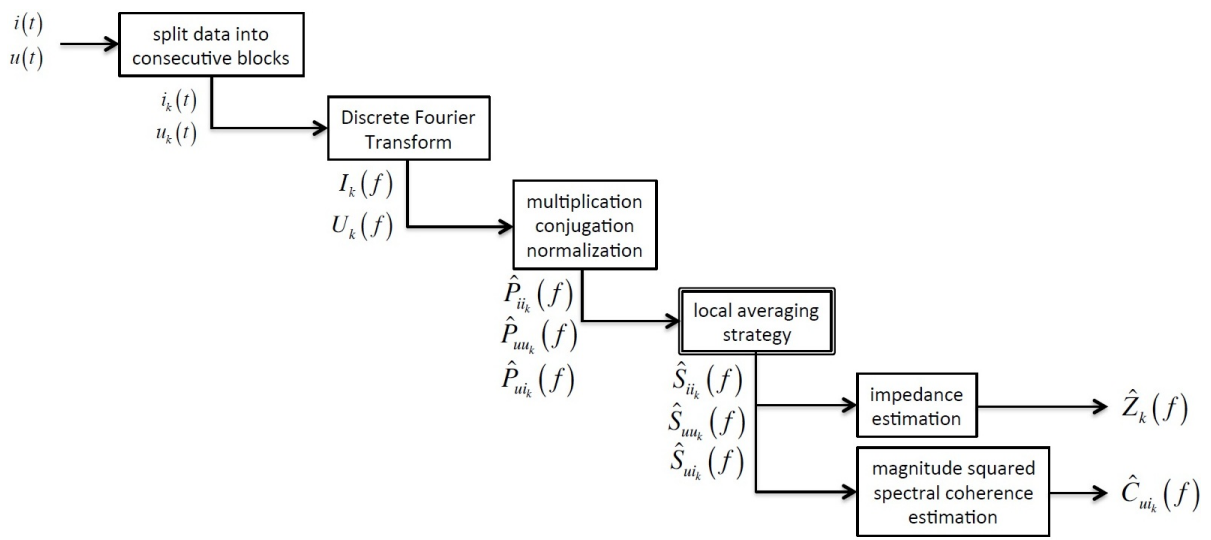

Figure 1: Structure of the time-varying estimation of the battery electrochemical impedance.

The main difference concerns the estimation of spectral quantities which are now locally averaged around each block of data with an exponential averaging strategy. The final expression of the impedance estimated for the block number $k$ is then:

$$
\hat{Z}_{k}(f)=\frac{\hat{S}_{u i_{k}}(f)}{\hat{S}_{i i_{k}}(f)},
$$

where the spectral quantities $\hat{S}_{u i_{k}}(f)$ and $\hat{S}_{i i_{k}}(f)$ are updated thanks to Eq. (7).

255 The obtained estimator is time-varying and updated at each new block of data in order to correctly estimate the true value of the battery impedance, and efficiently track its eventual variations over time. On the one hand, its tracking performance is related to the convergence time or similarly the response time of the averaging strategy: the smaller the convergence time is, the higher the tracking performance is. On the other hand, the estimation performance is directly related to the amount of residual variations after averaging, and therefore to the equivalent noise bandwidth of the averaging strategy: the lower the equivalent noise bandwidth is, the higher the estimation performance is. From expressions given in Table. 1, it is clear that the tradeoff between these two types of performance is controlled by the parameter $\alpha$ :

- When $\alpha$ is small and close to 0 , the convergence time is small leading to 
high tracking capabilities. But in this case, the equivalent noise bandwidth is high meaning poor averaging effect and poor estimation performance.

- On the contrary if $\alpha$ is high and close to 1 , the convergence time is large and the tracking capabilities decrease. However, the equivalent noise bandwidth is small in that case, leading to high averaging effect and good estimation performance.

Finally, Eq. (8) gives an interesting way to set the value of $\alpha$ : if the user wants the exponential averaging be equivalent to a sliding window of size $N, \alpha$ must be set by using this relation.

Following the same idea as for impedance estimation, the global estimator of the magnitude squared spectral coherence given in Eq. (5) can be adapted to the time-varying case by using local exponential averaging estimates of PSD and CPSD as shown in Eq. 10 .

$$
\hat{C}_{u i_{k}}(f)=\frac{\left|\hat{S}_{u i_{k}}(f)\right|^{2}}{\hat{S}_{u u_{k}}(f) \hat{S}_{i i_{k}}(f)} .
$$

The magnitude squared spectral coherence is then locally estimated around each block and averaged on an equivalent period of time controlled by the parameter $\alpha$ and Eq. (8).

In the two next sections, this algorithm is used and evaluated on simulated and experimental data.

\section{Experimental approach}

In this part, the time varying method of impedance estimation is tested on batteries. This study is performed on commercial lithium nickel manganese cobalt

The goal of this section is to validate the previous approach thanks to a battery simulator. This simulator models the behavior of the battery at $25{ }^{\circ} \mathrm{C}$. 
To model the system behavior, an electric model with a constant phase element (CPE) [18] 25] is used. The impedance of such an element is given by:

$$
Z_{\mathrm{cpe}}(w)=\frac{1}{Q(j w)^{p}},
$$

where $Q$ is a real constant, $j$ is a complex number such that $j^{2}=-1$ and $0 \leq p \leq 1$.

From Eq. (11), it is clear that if $p=1$ the CPE behaves as a simple capacitor whereas if $p=0$ it is considered as a resistor.

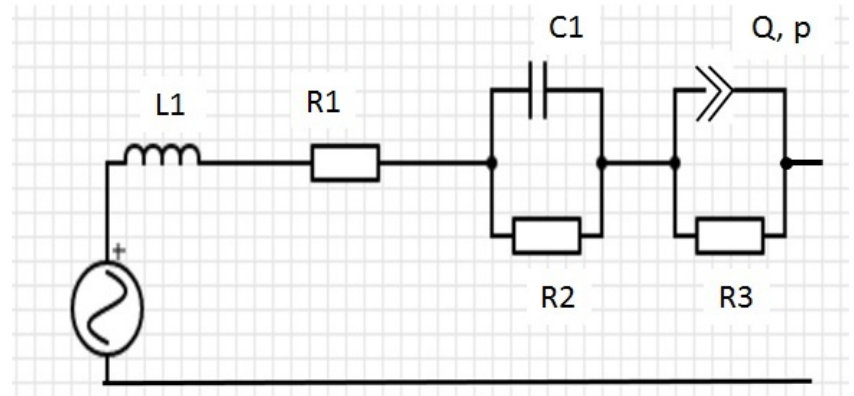

Figure 2: Equivalent electric model of the battery.

$$
Z_{\mathrm{thq}}(w)=R_{1}+j w L_{1}+\frac{R_{2}}{1+j w R_{2} C_{1}}+\frac{R_{3}}{1+R_{3} Q(j w)^{p}}
$$

Fig. 2 and Eq. 12 present the equivalent electric model of the battery. Each element is attributed to various physicochemical phenomena in the battery and the measurement chain [25] 26] 27]. Thus, $L_{1}$ corresponds to the inductance of connexions, $R_{1}$ is the purely ohmic resistance of the battery and the connexions, $R_{2}$ is linked to the charge transfer resistance and $C_{1}$ to the double layer capacity, $R_{3}$ and $Q, p$ model the diffusion of ions.

The value of each parameter is obtained with a Levenberg-Marquardt [23] 24] optimization algorithm applied to impedances estimated through narrowband FFT-EISs. These impedances are measured on a real battery under a constant discharge current of - 0.5 A from a SoC of $90 \%$ to $40 \%$ every $10 \%$ change. During this continuous discharge, parameters $L_{1}, C_{1}, Q, p$ stay constant whereas 
$R_{1}, R_{2}, R_{3}$ change. As an example, Table. 2 presents the value of each parameter obtained for a SoC of $90 \%$ and $40 \%$.

\begin{tabular}{|c|c|c|}
\hline Parameter & Value for the SoC $90 \%$ & Value for the SoC $40 \%$ \\
\hline$L_{1}$ & \multicolumn{2}{|c|}{$6.079^{*} 10^{-8} \mathrm{H}$} \\
\hline$C_{1}$ & \multicolumn{2}{|c|}{$1.173^{*} 10^{-1} \mathrm{~F}$} \\
\hline$Q$ & \multicolumn{2}{|c|}{$5.181 \mathrm{~F} . \mathrm{s}^{p-1}$} \\
\hline$p$ & \multicolumn{2}{|c|}{$6.02^{*} 10^{-1}$} \\
\hline$R_{1}$ & $4.648^{*} 10^{-2} \Omega$ & $4.768^{*} 10^{-2} \Omega$ \\
\hline$R_{2}$ & $3.541 * 10^{-3} \Omega$ & $4.352^{*} 10^{-3} \Omega$ \\
\hline$R_{3}$ & $1.359^{*} 10^{-2} \Omega$ & $1.340^{*} 10^{-2} \Omega$ \\
\hline
\end{tabular}

Table 2: Values of the model parameters obtained for a SoC of $90 \%$ and $40 \%$.

Fig. 3 clearly emphasis the variation of impedance during the discharge. It presents the Nyquist diagram of the modeled battery impedance for a SoC of $90 \%$ and $40 \%$, from 0.1 to $1000 \mathrm{~Hz}$ in blue, obtained from Eq. (12) and Table. 2. The red curves correspond to the frequency band of interest (from 20 to $90 \mathrm{~Hz}$ ) on which the time-varying method is tested in the experimental part. The magenta and green crosses are the real battery impedance values obtained from FFT-EIS for the same SoC, and used to check the validity of the modeled impedance. 


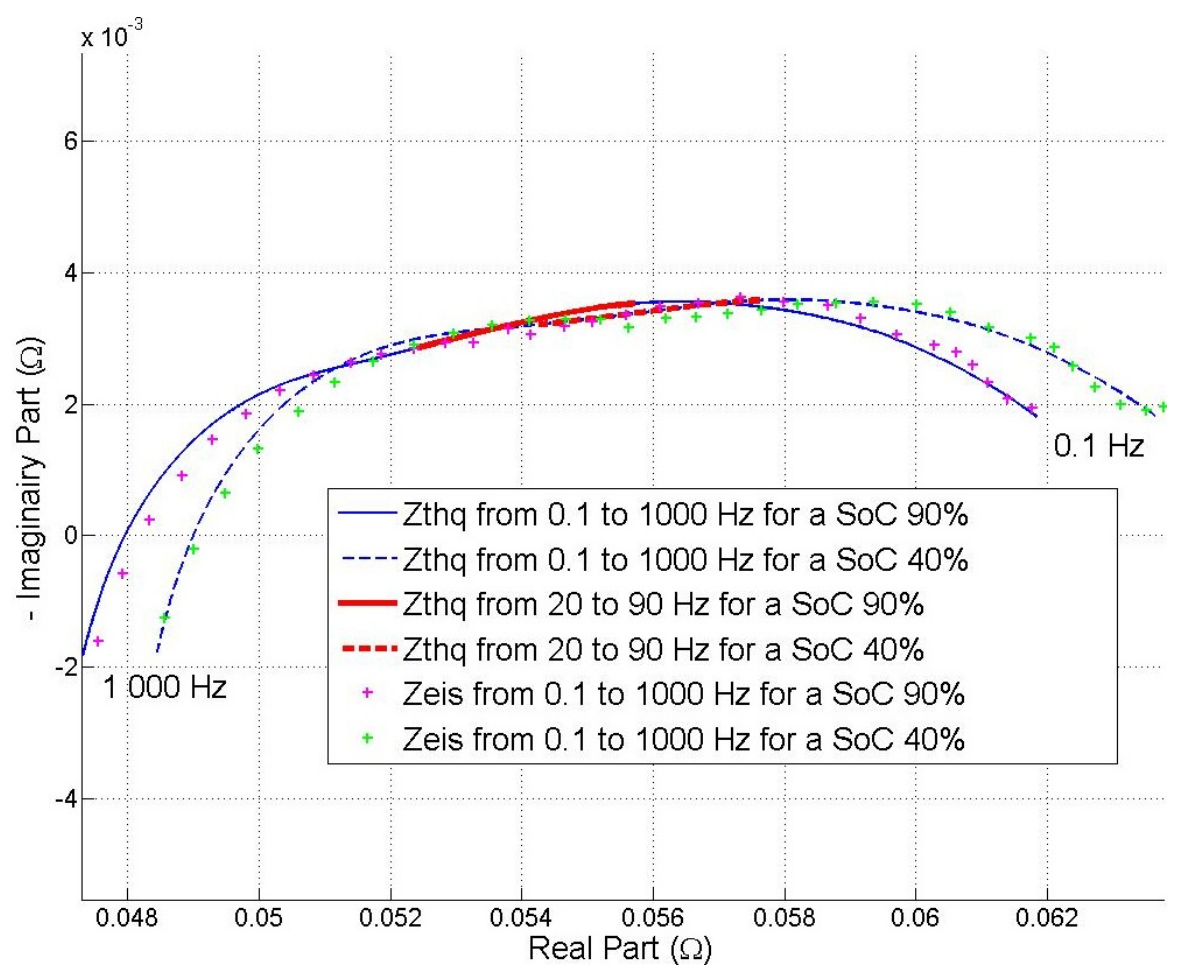

Figure 3: Nyquist diagram of the modeled and measured impedance for a SoC of $90 \%$ and $40 \%$.

\subsection{Simulation protocol}

with a constant polarization current of $-0.5 \mathrm{~A}$ which corresponds to a discharge ratio of $\frac{C}{4.4}$. A PRBS current is continuously added to this polarization current in order to perform impedance estimation and tracking. The amplitude of this additive PRBS signal is set to $250 \mathrm{~mA}$, which is sufficiently small for the battery to respect the linear assumption. In order to be in the same conditions as for the experimental results, the frequency band where the battery impedance is estimated is limited to $20 \mathrm{~Hz}-90 \mathrm{~Hz}$. This band is chosen because of instrumental constraints which are detailed in the experimental protocol. This frequency bandwidth corresponds to a PRBS block of length $0.25 \mathrm{~s}$. This block 
335 is then filtered thanks to a numeric low-pass filter with a cutoff frequency of $120 \mathrm{~Hz}$ to avoid abrupt variations in this additive current. The filtered block is then repeated continuously 31680 times, i.e. during $2.2 \mathrm{~h}$. The current and the voltage of the battery are, as in the experimental test, synchronously sampled at a sampling rate of $2500 \mathrm{~Hz}$. The simulated temperature is assumed constant

340 at $25^{\circ} \mathrm{C}$ during the discharge.

\subsection{Experimental protocol}

The battery is placed in an enclosure with controlled temperature of $25^{\circ} \mathrm{C}$. After a complete charge $\left(25^{\circ} \mathrm{C}\right.$, constant current of $\frac{C}{2}$ until $4.2 \mathrm{~V}$, constant voltage during $1 \mathrm{~h}$ ), we discharge the battery at a current of $-0.5 \mathrm{~A}$ which corre345 sponds to a rate of discharge of $\frac{C}{4.4}$.

The estimates obtained by the time-varying wideband frequency algorithm is compared to a known reference impedance called $Z_{r e f}(f)$. This reference is measured through an impedance spectroscopy thanks to a potentiotat VMP3 of Bio-Logic SAS with a booster 5A, associated with the EC-Lab software (signal amplitude of $200 \mathrm{~mA}, 1 \mathrm{~Hz}$ to $1 \mathrm{kHz}$, logarithm spacing of 10 measures per decade, 3 measures per frequency). Therefore, once $10 \%$ of SoC is discharged, we first record a classical FFT-EIS in a galvanostatic mode at the polarization current. For instance, Fig. 3 presents the Nyquist diagram of this NMC battery obtained from the FFT-EIS for the SoC $90 \%$ and $40 \%$.

355 Then we consecutively apply the same block of filtered PRBS block, which was described in the protocol of the simulation, centered again around -0.5 $\mathrm{A}$ in order to apply global (Eq. (3b) and exponential averaging and compare the obtained results. This low polarization current allows to minimize the SoC variation during the measurement time.

360 This filtered block is repeated consecutively 36 times, leading to a measurement process with a total duration of $9 \mathrm{~s}$. During this process, the current and the voltage of the battery are synchronously sampled at a rate of $2500 \mathrm{~Hz}$. The bandwidth studied begins at Fmin $=20 \mathrm{~Hz}$ and closes at Fmax $=90 \mathrm{~Hz}$, due to instrumental constraints. 

cations. This clearly limits the measurement time and consequently the lower frequency at which the impedance can be correctly estimated. Indeed both the range of the sampling frequency and the number of points of an acquisition are limited. The lower Fmin is, the longer the SBPA block is. By choosing Fmax,

370 the bit frequency Fbit of the PRBS block is defined as Fbit $=\frac{F \max }{0.4}[14$ and the sampling frequency $\mathrm{Fe}$ as at least $F e=10 \mathrm{Fbit}$. To raise Fmax, the sampling frequency should also be increased, yet for our experiment the sampling frequency is limited at most to $5 \mathrm{kHz}$. Moreover the higher the sampling frequency is, the larger the memory is. an embedded measurement system. This frequency band corresponds approximatively to the end of the first semicircle on the Nyquist diagram of the battery impedance. This area is closely related to the contributions of both ohmic and charge transfer resistance of the battery, which is an important parameter related to common state indicators such as the SoC and the SoH. Being able to estimate the battery impedance in this particular frequency band thus provides an efficient way to estimate this parameter and obtain information on the battery SoC and SoH. Indeed in the chosen frequency band, the battery impedance varies significantly with respect to the SoC, justifying the development of an 385 evolutionary method. This point is highlighted by Fig. A in complementary information.

\section{Results and discussion}

\subsection{Simulation results}

The time-varying impedance spectroscopy method developed in section 2 and summarized in Fig. 1 is applied to the simulated signals for the frequency band of interest $(20 \mathrm{~Hz}-90 \mathrm{~Hz})$. A Hann window and a forgetting factor $\alpha=0.9$ are chosen to estimate the necessary PSDs and CPSD with Eq. (7), the time-varying impedance with Eq. (9) and the time-varying magnitude squared 
spectral coherence with Eq. 10 . This forgetting factor value corresponds to a local averaging over $N=19$ blocks (see Eq. (8)) or equivalently a local averaging time of $4.75 \mathrm{~s}$, and a response time at $80 \%$ of $3.56 \mathrm{~s}$ (see Table. 1).

After this transient period, the algorithm is in steady state and the estimations can be considered as valid. On the frequency band of interest, the magnitude squared spectral coherence stays above 0.99 all along the simulation. This confirms that the battery can be considered as a LTI system during the equivalent averaging time, and that its electrochemical impedance can be correctly estimated. Fig. 4 presents the Bode diagram of the estimated impedance obtained with these settings for different $\mathrm{SoC}$ values during the whole discharge. After each decrease of $10 \%$ of SoC, the estimated impedance is compared to its theoretical value computed thanks to Eq. (12).
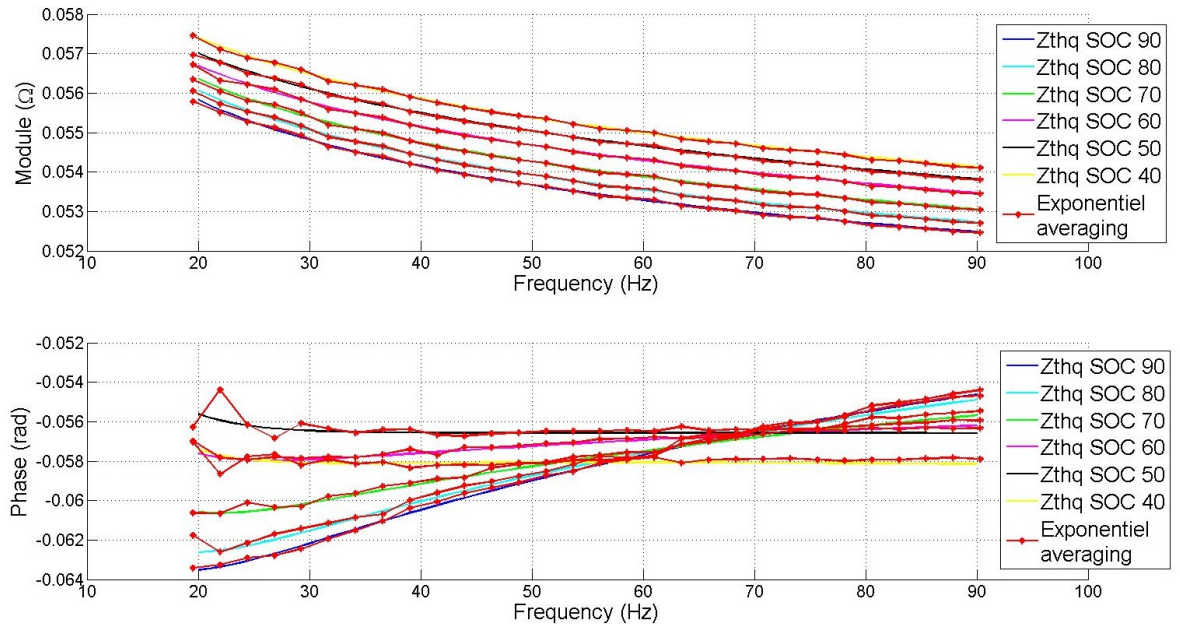

Figure 4: Bode diagram of the estimated impedance during a discharge for different SoC values (top: modulus; bottom: phase).

Fig. 4 shows that whatever the SoC value, the estimated impedance obtained 
with the proposed method is very close to its theoretical value. The relative bias averaging estimation is very close to the estimation obtained through the FFTEIS. However the exponential averaging method seems less accurate. This can 
be explained by the time window over which each estimator is averaged. The global averaging estimation is averaged over the whole data set ( $N=36$ blocks) leading to good estimation performance. On the contrary, the exponential averaging estimation is averaged over a smaller local window $(\alpha=0.9, N=19$ blocks) whose size is set by using the forgetting factor $\alpha$ (the closer to $1 \alpha$ is, the longer the time window is).
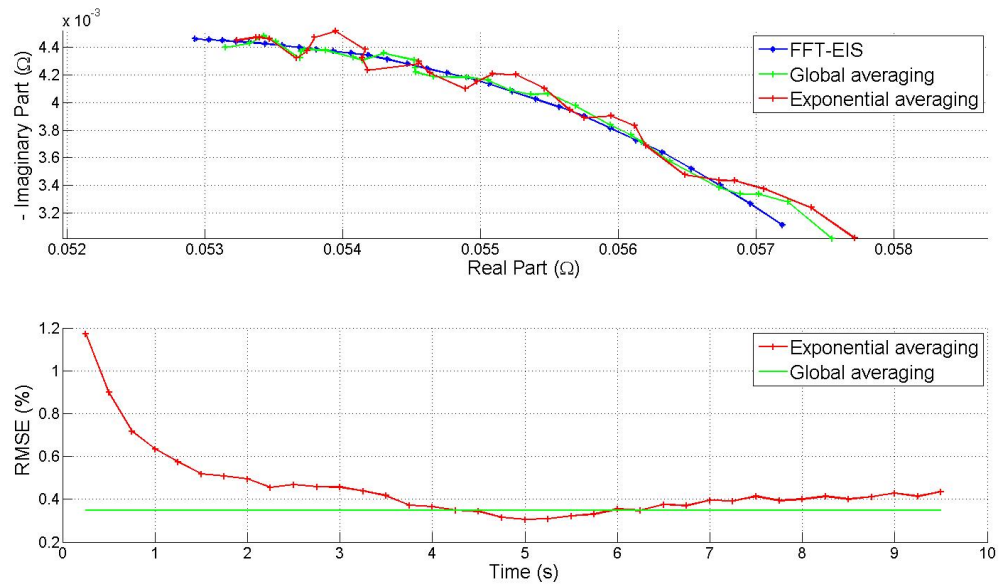

Figure 5: Estimated impedances between $20 \mathrm{~Hz}$ to $90 \mathrm{~Hz}$ by using the three methods (top: Nyquist diagram for exponential averaging $\alpha=0.9$ (red), global averaging (green), and the EIS method (blue), bottom: error curves (time evolution of the root-mean-square error)).

The error curves shown in Fig. 5 represent the time evolution of the estimation performance indicator defined in the exponential (red curve) and global (green curve) cases. This indicator corresponds to the normalized root-meansquare error (RMSE Eq. (13) ) between a known reference value of the impedance $Z_{r e f}(f)$ and the $k^{\text {th }}$ estimated value of this impedance $Z_{k}(f)$ described in Eq. (13). As expected, the error for the global averaging algorithm is always lower than the one obtained in the exponential averaging case. Moreover two 
steps are clearly visible on the red error curve: a first decaying part corresponding to the convergence time at $80 \%$ of the algorithm (duration $=3.56 \mathrm{~s}$ for $\alpha=0.9)$, and a second part after convergence where the estimate fluctuates around the correct impedance. We can see the same two steps on the film of the discharge on the simulator, after the convergence time, the estimation is really more accurate.

$$
\operatorname{RMSE}_{k}=100 \sqrt{\frac{\sum_{f \in B}\left|Z_{r e f}(f)-\hat{Z}_{k}(f)\right|^{2}}{\sum_{f \in B}\left|Z_{r e f}(f)\right|^{2}}}
$$
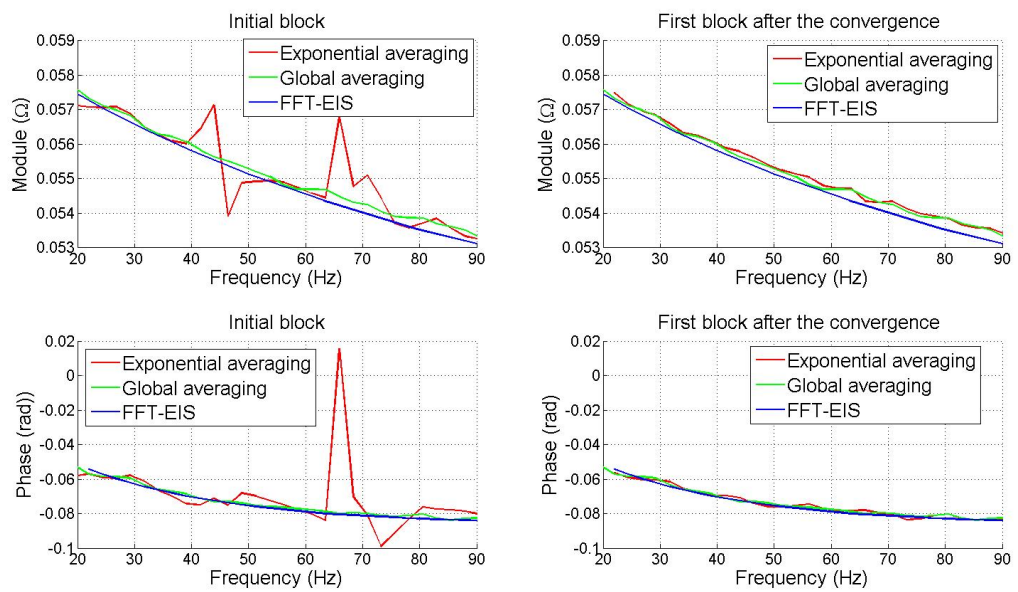

Figure 6: Difference between the initial estimation and the first estimation after the convergence for the exponential case (left: $1^{\text {st }}$ estimation at time $\mathrm{t}=0.25 \mathrm{~s}$, right: estimation after the convergence at time $\mathrm{t}=3.56 \mathrm{~s}$, top: module, bottom: phase).

Fig. 6 underlines the difference of estimation accuracy between the first and after convergence temporal estimates of the battery impedance (both module and phase are represented). Once the convergent step of the exponential averaging algorithm is finished, the estimation performance becomes clearly more accurate, explaining the large decrease in the error curve of Fig. 5. The parameter which controls the length of the convergence time and the quantity of 
fluctuations around the final error is the forgetting factor $\alpha$. Fig. 7 highlights the influence of this parameter on the estimated impedance (Nyquist diagrams) and on the learning curve of the algorithm. For a forgetting factor close to 1 ( $\alpha=0.9, N=19$ blocks, red curves), a large convergence time is obtained (around $3.56 \mathrm{~s}$ ) with small final estimation error and fluctuations. For a smaller forgetting factor ( $\alpha=0.5, N=3$ blocks according to Eq. (8), black curves), the convergence time is much shorter (around $0.33 \mathrm{~s}$ ) but the final estimation error and fluctuations are increased.
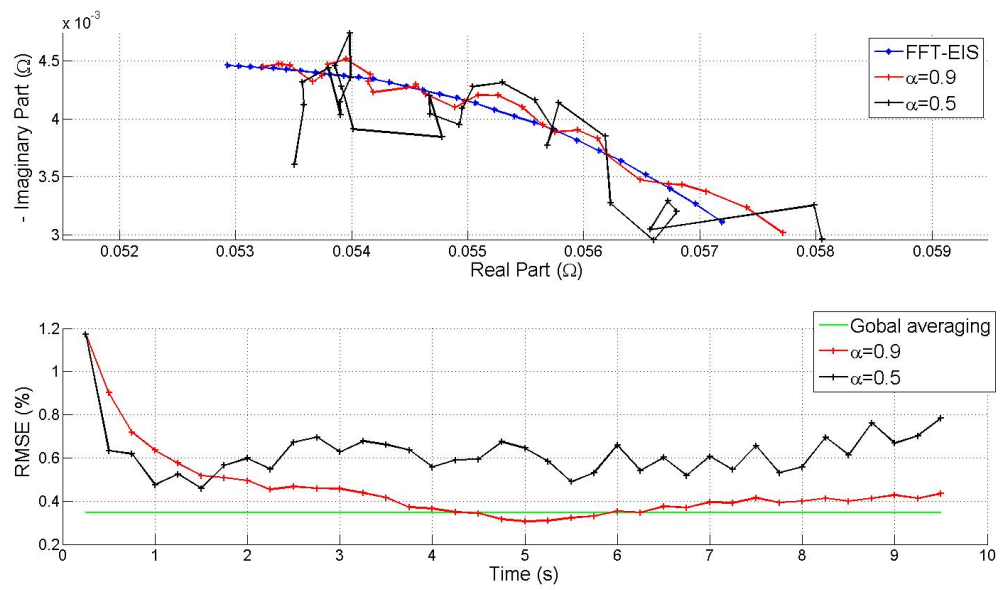

Figure 7: Influence of the forgetting factor (top: Nyquist diagram for $\alpha=0.9$ (red), $\alpha=0.5$ (black), and the EIS method (blue), bottom: error curves for $\alpha=0.5$ (black), $\alpha=0.9$ (red), and the sliding window algorithm (green)).

These results emphasis the existence of a trade-off between the obtained convergence time (related to the tracking capabilities of the algorithm) and the final estimation error (related to the estimation performance of the algorithm). This trade-off can be managed through the value of the forgetting factor $\alpha$. Indeed, a small forgetting factor leads to an algorithm able to follow strong variations of the estimated impedance over time, but with a large estimation error. On the 
contrary, a forgetting factor close to 1 leads to an algorithm able to precisely estimate the battery impedance, but unable to follow large time variations.

To check the repeatability of the time-varying method, the experiment was repeated during 5 charges discharges cycles. The repeatability of the exponential method was evaluated and compared to the FFT-EIS performances.

In a first part, the aim is to quantify the repeatability of the FFT-EIS. For each SoC, 5 FFT-EISs estimations are averaged according to Eq. (14) and the standard deviation defined by Eq. (15) is calculated. Fig. 8 presents on a Bode diagram the averaged module and phase for several SoC.

$$
\begin{gathered}
Z_{m}^{\text {eis }}(f, S o C)=\frac{1}{5} \sum_{i_{\text {dech }}=1}^{i_{\text {dech }}=5} Z_{i_{\text {dech }}}^{\text {eis }}(f, S o C) . \\
Z_{\text {std }}^{\text {eis }}(f, S o C)=\sqrt{\frac{1}{4} \sum_{i_{\text {dech }}=1}^{i_{\text {dech }}=5}\left|Z_{i_{\text {dech }}}^{\text {eis }}(f, S o C)-Z_{m}^{\text {eis }}(f, S o C)\right|^{2} .}
\end{gathered}
$$

495 where $Z_{i_{\text {dech }}}^{\text {eis }}(f, S o C)$ is the impedance estimator for one $\mathrm{SoC} ; Z_{m}^{\text {eis }}(f, S o C)$ corresponds to averaged impedance and $Z_{\text {std }}^{\text {eis }}(f, S o C)$ its standard deviation. On the module part (on Fig. 8), it is very easy to distinguish each SoC, whereas it is more difficult on phase because of the inflection point around $25 \mathrm{~Hz}$. 

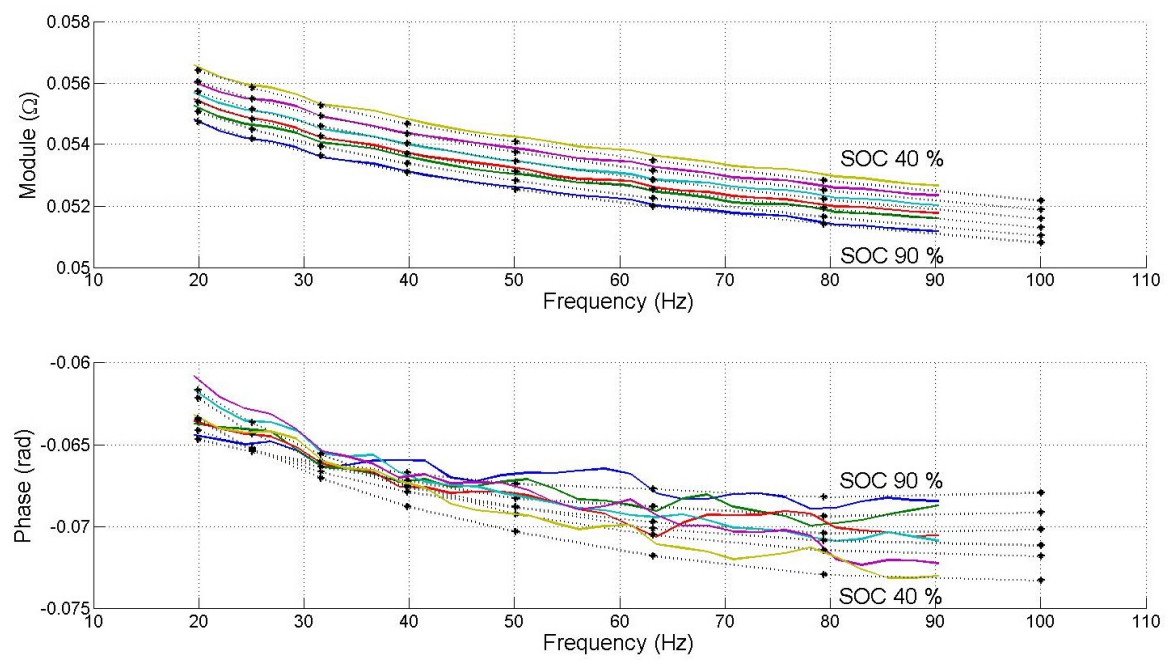

Figure 8: Bode diagram of the average on 5 discharges of impedance at 6 SoC (top: Module; bottom: Phase).

The relative standard deviation average on the frequency bandwidth of the module is lower than the one of the phase, however in both cases these quantities are under $1.2 \%$, which show the good repeatability of this method. After having quantify the repeatability of the FFT-EIS, the tracking method is tested in a second part.

For each SoC, once convergence time is past, 5 estimations obtained with the exponential method are averaged by Eq. (16) and the standard deviation is calculated by Eq. 17).

$$
\begin{gathered}
Z_{m}(f, S o C)=\frac{1}{5} \sum_{i_{\text {dech }}=1}^{i_{\text {dech }}=5} \bar{Z}_{i_{\text {dech }}}(f, S o C) . \\
Z_{\text {std }}(f, S o C)=\sqrt{\frac{1}{4} \sum_{i_{\text {dech }}=1}^{i_{\text {dech }}=5}\left|\bar{Z}_{i_{\text {dech }}}(f, S o C)-Z_{m}(f, S o C)\right|^{2}} .
\end{gathered}
$$

where $\bar{Z}_{i_{\text {dech }}}(f, S o C)$ is the temporal average of impedance estimator for all the estimations after the convergence time for one SoC; $Z_{m}(f, S o C)$ corresponds to averaged impedance on 5 cycles and $Z_{s t d}(f, S o C)$ its standard deviation. 
The results are presented on Bode diagram in Fig. 8 for several SoC. If on the module part it is again easy to follow the impedance evolution during the discharge, the phase presents more variations at some frequencies. Again an inflection point can be seen but with more difficulty than one in the FFT-EIS case because of the noise on the estimates.

The phase and module relative bias averages on the frequency bandwidth between the estimation by the proposed method and the FFT-EIS are calculated. For each SoC the relative bias of the module (phase respectively) is under $1 \%$ (respectively $2 \%$ ). The relative standard deviation average on the frequency bandwidth is under $0.1 \%$ for the module and $1.1 \%$ for the phase. These relative standard deviations are of the same order of magnitude than FFT-EIS. 525 The battery impedance was not estimated for extreme SoC values of $100 \%$ and below $10 \%$. For a SoC of $100 \%$, the main reason is that measurement processes were carried on only during battery discharges. Under these conditions, the estimation of the battery impedance with a SoC of $100 \%$ presents no practical interest and was not considered in the paper. For SoC values below $10 \%$, technical difficulties are encountered to estimate the battery impedance. Indeed, the results obtained with the proposed method are compared to those obtained with FFT-EIS method. Unfortunately, no security system prevents the battery from deterioration at the end of the discharge during FFT-EIS. The impedance estimation for SoC $40 \%$ to $10 \%$ is supplied as supplementary in535 formation (Fig. B).

On this study over 5 discharges of the lithium-ion battery, the exponential method presents for each SoC low relative standard deviations and bias. The exponential method can so be considered as repeatable as the FTT-EIS method. 
More and more applications use batteries. The development of BMS is needed to optimize their efficiency and dependability. With this in mind, estimate in real time the electrochemical impedance temporal evolution is a valuable tool. The method presented in this paper provides one solution to this open problem.

This method not only allows an accurate estimation of the battery impedance, but also a tracking of its temporal evolution. In other words, the developed method presents the major advantage to be evolutionary and to follow the temporal evolution of the impedance compared to classical estimation methods like FFT-EIS. It mainly relies on a simple exponential averaging strategy, and allows to manage the trade-off between its tracking and estimation performance through a single normalized parameter: the forgetting factor.

The chosen exponential averaging strategy can be recursively implemented with a low memory depth. Moreover, the use of a wideband signal such as PRBS can be generated with very simple electronics circuits. These two particularities clearly show that the proposed method is particularly relevant for embedded applications.

The proposed estimator was tested on both a simulated and real lithium ion battery, because this technology is widely used for electric or hybrid transports or connected objects. However, this method is not limited to lithium ion batteries and could also be applied to any other electrochemical systems such as primary battery, secondary battery (including aqueous technology or other) or fuel cells .

On the simulator, the exponential averaging method allows to track in real time the temporal variations of electrochemical impedance. The obtained results demonstrate the capabilities of the method to precisely track the battery impedance evolution over more than $50 \%$ of SoC discharge.

On the real battery we checked the influence of the forgetting factor on the performance of estimation. A trade-off between the tracking and estimation per- 
that the proposed method finally appears as repeatable as the FFT-EIS method. The proposed method provides not only an accurate and repeatable estimation of the impedance but also a tracking of its fluctuations over time. In further work, this algorithm will be embedded, applied on a test bench, and the developed method will be tested on batteries of different chemistries.

\section{References}

[1] M. Coleman, C. K. Lee, C. Zhu, W. Hurley, State-of-charge determination from emf voltage estimation: Using impedance, terminal voltage, and current for lead-acid and lithium-ion batteries, IEEE TRANSACTIONS ON

[2] D. Andrea, Battery Management Systems for Large Lithium Ion Battery Packs, Artech House, 2010.

[3] D. Howey, V. Yufit, P. Mitcheson, G. Offer, N. Brandon, Impedance measurement for advanced battery management system, EVS27 Internationale

[4] W. Waag, S. Kbitz, D. Sauer, Experimental investigation of the lithiumion battery impedance characteristic at various conditions and aging states and its influence on the application, Applied Energy 102 (2013) 885-897.

[5] J. Zhu, Z. SUN, X. Wei, H. Dai, A new lithuim-ion battery internal temperature in-line estimate method based on electrochemical impedance spectroscopy measurement, Journal of Power Sources 274 (2015) 990-1004.

[6] R. Richardson, P. Ireland, D. Howey, Battery internal temperature estimation by combined impedance and surface temperature measurement, Journal of Power Sources 63 (2014) 254-261. 
[7] J. Schmid, S. Arnold, A. Loges, D. Werner, T. Wetzel, E. Ivers-Tiffe, Measurement of the internal cell temperature via impedance: Evaluation and application of a new method, Journal of Power Sources 243 (2013) 110-117.

[8] K. Bundy, M. Karlsson, G. Lindbergh, A. Lundqvist, An electrochemical impedance spectroscopy method for prediction of the state of charge of a nickel metal battery at open circuit and during discharges, Journal of Power Sources 72 (1998) 118-125.

[9] S. Rodrigues, N. Munichandraiah, A. Shukla, A review of state-of-charge indication of batteries by means of a.c. impedance measurements, Journal of Power Sources 87 Issue 1-2 (2000) 12-20.

[10] H. Blanke, O. Bohlen, S.Buller, R. Doncker, B. Fricke, A. Hammouche, D. Linzen, M. Thele, D. Sauer, Impedance measurements on leadacid batteries for state-of-charge, state-of-health and cranking capability prognosis in electric and hybrid electric vehicles, Journal of Power Sources 144 Issue 2 (2005) 418-425.

[11] U. Troltzsch, O. Kanoun, H. Trankler, Characterizing aging effects of lithium ion batteries by impedance spectroscopy, Electrochemica Acta 51 Issue 8-9 (2006) 1664-1672.

[12] L. Ljung, System Identification - Theory for the User, 2nd Edition, Prentice Hall, 1999.

[13] K. Shin, J. Hammond, Fundamentals of Signal Processing for Sound and Vibration Engineers, 2008.

[14] R. Pintelon, J. Schoukens, System Identification - a Frequency Domain Approach, 2nd Edition, Wiley, 2012.

[15] J. S. Bendat, A. G. Piersol, Random Data - Analysis and Measurement Procedures, 4th Edition, Wiley, 2010. 
[16] R.Al-Nazer, V. Cattin, P. Granjon, Broadband identification of battery electrical impedance for hevs, IEEE Transactions on Vehicular Technology 62 n. 7 (2013) 2896-2905.

[17] F. Huet, A review of impedance measurements for determination of the state-of-charge or state-of-health of secondary batteries, Journal of Power Sources (1998) 59-69.

[18] E. Barsoukov, J. R. Macdonald, Impedance Spectroscopy - Theory, Experiment, and Applications, 2nd Edition, Wiley, 2005.

[19] P.-D. Welch, The use of fast fourier transform for the estimation of power spectra: A method based on time averaging over short, modified periodograms, IEEE Trans. on Audio Electroacoustics, Vol. AU-15 (1967) 7073.

[20] D. Howey, P. Mitcheson, V. Yufit, G. Offer, N. Brandon, On-line measurement of battery impedance using motor controller excitation, Vehicular Technology, IEEE Transactions 63 (2014) 1664-1672.

[21] A. Lasia, Electrochemical Impedance Spectroscopy and its Applications, Springer-Verlag New York, 2014.

[22] T. Yokoshima, D. Mukoyama, K. Nakazawa, Y. Gima, H. Isawa, H. Nara, T. Momma, T. Osaka, Application of electrochemical impedance spectroscopy to ferri/ferrocyanide redox couple and lithium ion battery systems using a square wave as signal input, Electrochemica Acta 180 (2015) 922-928.

[23] K. Levenberg, A method for the solution of certain non-linear problems in least squares, Quaterly of Applied Mathematics 2 (1944) 164-168.

[24] D.Marquardt, An algorithm for least squares estimation of nonlinear parameters., SIAM Journal on Applied Mathematics 11 (1963) 431-441. 
[25] M. Orazem, B. Tribollet, Electrochemical Impedance Spectroscopy, Prentice Hall, 2011.

[26] T. Dong, A. Kirchev, F. Mattera, J. Kowal, Y. Bultel, Dynamic modeling of li-ion batteries using an equivalent electrical circuit, Journal of The Electrochemical Society 72 .

[27] A. Jossen, Fundamentals of battery dynamics, Journal of Power Sources (2006) 530-538. 